\section{Miniature wideband bandpass filter based on electric and magnetic coupling paths}

\section{H. Asadbeigi and B.S. Virdee}

A compact wideband bandpass filter design that provides a sharp rolloff and low loss performance is presented. The design is based on employing two separate paths between the adjacent resonators constituting the filter, where one path enables electric coupling and the other one magnetic coupling. This structure generates zeros in the filter's transmission response due to the dominance of electric coupling. A fourth-order filter was fabricated and tested. Correlation between the simulated and measured results is remarkable. The measured results confirm the fractional bandwidth of $70 \%$ at the centre frequency of $4.58 \mathrm{GHz}$, insertion-loss is $<0.55 \mathrm{~dB}$ with a return-loss better than $15 \mathrm{~dB}$ in the passband. The filter occupies an area of $4.1 \times 17.8 \mathrm{~mm}$, which corresponds to $0.1 \lambda_{\mathrm{o}} \times 0.44 \lambda_{\mathrm{o}}$, where $\lambda_{\mathrm{o}}$ is the wavelength of the centre frequency.

Introduction: Wideband bandpass filters (WBFs) are essential for high data throughput and high-speed connectivity in modern wireless communication systems. The bandwidth of conventional filters can be increased by simply increasing the coupling between the adjacent resonators, and this generally improves its insertion-loss (IL) performance. This can be achieved by reducing the interval between the resonators; however, the gap requirement in most cases is not practically feasible. Alternatively the bandwidth can be increased by increasing the order of the filter, but this tends to increase the filter's IL and physical size. Hence, various methods and techniques have been investigated previously to overcome these limitations. In [1-3], the authors present a multimode operation WBF using stub perturbation, a folded resonator and a symmetric resonator. Reported in [4] is a broadband bandpass filter constructed using the Y-shaped resonator with I/O cross-coupling that operates in a dual mode. The transversal signal interaction concept and a T-shaped resonator were employed to design the WBF in [5]. The shortfall of the above techniques is that the circuit size is still considered relatively large for many applications, and the improvement in bandwidth is restricted. To address these disadvantages, in this Letter a WBF is described based on a separate electric and magnetic coupling paths topology. Second- and fourth-order WBFs were investigated. The results show that the proposed filter topology enhances the filter's selectivity by generating transmission zeros in the region below the passband, when the electric coupling is dominant. Finally, a fourth-order WBF was fabricated and tested.
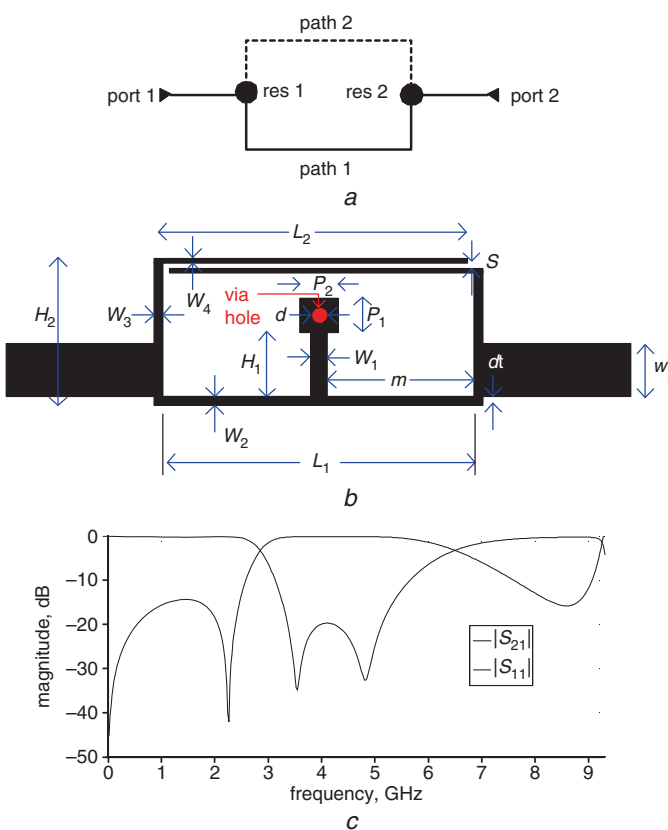

Fig. 1 Topology, layout and responses of second-order WBF

a Topology

$b$ Layout

c Simulated $S$-parameters
Filter design: Fig. $1 a$ depicts the topology of the proposed WBF for a second-order design. In this Figure, two coupling paths are available between resonators 1 and 2, where path 1 and path 2 provide magnetic and electric coupling, respectively. Fig. $1 b$ shows the layout of the proposed second-order WBF, where the open-end parallel coupled transmission line provides electric coupling and the common shorted transmission line between the two resonators provides magnetic coupling. Depending on the dimensions of the coupling sections, either electric or magnetic coupling can be dominant, and here the electric coupling is made to be dominant. Fig. $1 c$ shows simulated $S$-parameters where a transmission zero is located at $2.26 \mathrm{GHz}$ (lower edge of the passband) with a rejection of $41.9 \mathrm{~dB}$. In addition, the IL is $<0.25 \mathrm{~dB}$ between 3.54 and $5.21 \mathrm{GHz}$ and the return loss is better than $20 \mathrm{~dB}$ in the passband. The simulated results were carried out with IE3D software. The dimensions of Fig. $1 b$ are: $d \mathrm{t}=0.3 \mathrm{~mm}$, $L_{1}=8.57 \mathrm{~mm}, W_{1}=0.54 \mathrm{~mm}, H_{1}=1.72 \mathrm{~mm}, P_{1}=1 \mathrm{~mm}, P_{2}=1 \mathrm{~mm}$, $d=0.5 \mathrm{~mm}, \quad m=4.02 \mathrm{~mm}, \quad W_{2}=0.24 \mathrm{~mm}, \quad H_{2}=4.08 \mathrm{~mm}, \quad W_{3}=$ $0.22 \mathrm{~mm}, W_{4}=0.09 \mathrm{~mm}, L_{2}=8.48 \mathrm{~mm}, S=0.08 \mathrm{~mm}$ and $w_{f}=1.3 \mathrm{~mm}$.

Fig. $2 a$ shows the topology of the fourth-order WBF. Two (electrical and magnetic) coupling paths exit between resonators 1 and 2 . In addition, two (electrical and magnetic) coupling paths exit between resonators 3 and 4 , and one coupling path exits between resonators 2 and 3 . The layout of the proposed fourth-order WBF is shown in Fig. $2 b$, where a second-order WBF is combined with an identical inverted second-order WBF. Fig. $2 c$ shows simulated $S$-parameters where transmission zeros exit at 1.82 and $2.76 \mathrm{GHz}$ with rejection levels of 69.1 and $38.7 \mathrm{~dB}$. In addition, the simulated IL is $<0.44 \mathrm{~dB}$ in the passband.
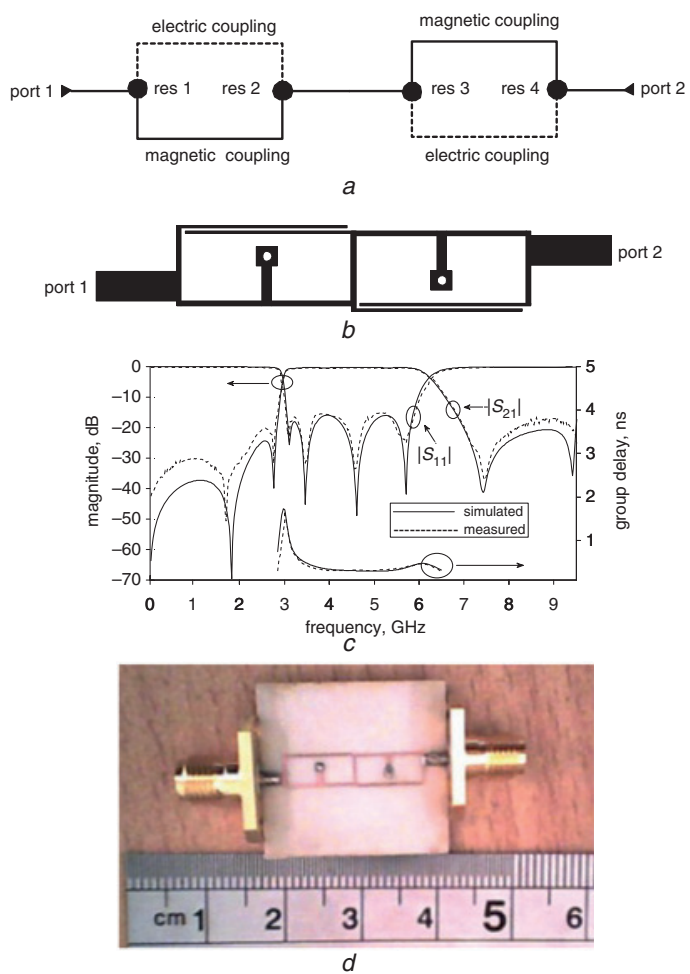

Fig. 2 Topology, layout, responses, and photograph of fourth-order WBF a Topology

$b$ Layout

c Simulated and measured responses

$d$ Photograph

Simulated and experimental results: The proposed filter was fabricated on RO4003C with a substrate thickness of $0.508 \mathrm{~mm}$, permittivity of 3.38 and loss tangent of 0.0027 , as shown in Fig. $2 d$. Fig. $2 c$ shows the simulated and measured results of $S$-parameters and group delay. The filter's $3 \mathrm{~dB}$ bandwidth is from 2.98 to $6.19 \mathrm{GHz}$, with a centre frequency of $4.58 \mathrm{GHz}$ and compact size of $4.08 \times 17.8 \mathrm{~mm}$. The measured IL is $<0.55 \mathrm{~dB}$, whereas the return loss is better than $15 \mathrm{~dB}$. The out-of-band rejection is $20 \mathrm{~dB}$ from $\mathrm{DC}$ to $2.8 \mathrm{GHz}$ and $17 \mathrm{~dB}$ from 6.9 to $9.5 \mathrm{GHz}$. A comparison with other recently reported WBFs is given in Table 1, which shows that the proposed design has a bigger fractional bandwidth, lower loss and is significantly smaller. 
Table 1: Performance comparison of proposed filter with other recently published works

\begin{tabular}{|c|c|c|c|c|c|}
\hline Refs. & $f_{0}(\mathrm{GHz})$ & $3 \mathrm{~dB}$ FBW $(\%)$ & IL $(\mathrm{dB})$ & Circuit size $\left(\lambda_{\mathrm{o}} \times \lambda_{\mathrm{o}}\right)$ & $N$-size \\
\hline$[1]$ & 5.15 & 45.0 & 1.20 & $0.85 \times 0.32$ & 6.18 \\
\hline$[2]$ & 5.30 & 60.0 & - & $0.84 \times 0.36$ & 6.87 \\
\hline$[3]$ & 7.00 & 62.7 & 1.65 & $0.62 \times 0.48$ & 6.76 \\
\hline$[4]$ & 4.30 & 65.0 & 0.50 & $0.41 \times 0.39$ & 3.63 \\
\hline$[5]$ & 4.00 & 36.3 & 1.40 & $0.68 \times 0.39$ & 6.02 \\
\hline$[6]$ & 3.01 & 27.0 & 0.20 & $0.61 \times 0.28$ & 3.88 \\
\hline$[7]$ & 1.45 & 57.9 & 1.00 & $0.15 \times 0.64$ & 2.18 \\
\hline This work & 4.58 & 70.0 & 0.55 & $0.10 \times 0.44$ & 1.00 \\
\hline
\end{tabular}

IL: insertion loss; $N$-size: normalised size compared with size of proposed filter

Conclusion: A novel WBF design is described. The proposed filter exhibits the desirable features of high selectivity, low loss, a flat passband response, a simple structure, wide passband response and compact size. The measured results validate the filter characteristics.

(C) The Institution of Engineering and Technology 2014

15 March 2014

doi: $10.1049 / \mathrm{el} .2014 .0927$

One or more of the Figures in this Letter are available in colour online.

H. Asadbeigi (Department of Electronics, Toyserkan Branch, Islamic Azad University, Toyserkan, Iran)

E-mail: hamid.asadbeigi@yahoo.com
B.S. Virdee (Faculty of Life Sciences and Computing, Centre for Communications Technology, London Metropolitan University, London, United Kingdom)

\section{References}

1 Ma, K., Liang, K.C.B., Jayasuriya, R.M., and Yeo, K.S.: 'A wideband and high rejection multimode bandpass filter using stub perturbation', IEEE Microw. Wirel. Compon. Lett., 2009, 19, (1), pp. 24-26

2 Wang, H., Chu, Q.-X., and Gong, J.-Q.: 'A compact wideband microstrip filter using folded multiple-mode resonator', IEEE Microw. Wirel. Compon. Lett., 2009, 19, (5), pp. 287-289

3 Feng, W.J., Zhou, J.G., and Che, W.Q.: 'Wideband bandpass filter using symmetric multimode resonator', Electron. Lett., 2012, 48, (17), pp. 1071-1073

4 Song, K., and Xue, Q.: 'Novel broadband bandpass filters using Y-shaped dual-mode microstrip resonators', IEEE Microw. Wirel. Compon. Lett., 2009, 19, (9), pp. 548-550

5 Feng, W., Che, W., Shi, S., and Xue, Q.: 'High selectivity wideband bandpass filter based on transversal signal-interaction concepts and T-shaped structure', IEEE Microw. Wirel. Compon. Lett., 2012, 22, (11), pp. 562-564

6 Chen, F.-C., Qiu, J.-M., Chen, Z.-H., and Chu, Q.-X.: 'Low insertion loss wideband bandpass filter with six transmission zeros', Electron. Lett., 2013, 49, (7), pp. 477-479

7 Fan, J., Zhan, D., Jin, C., and Luo, J.: 'Wideband microstrip bandpass filter based on quadruple mode ring resonator', IEEE Microw. Wirel. Compon. Lett., 2012, 22, (7), pp. 348-350 